\title{
Pressure relieving support surfaces (PRESSURE) trial: cost effectiveness analysis
}

Cynthia Iglesias, Jane Nixon, Gillian Cranny, E Andrea Nelson, Kim Hawkins, Angela Phillips, David Torgerson, Su Mason, Nicky Cullum, on behalf of the PRESSURE Trial Group

\begin{abstract}
Objective To assess the cost effectiveness of alternating pressure mattresses compared with alternating pressure overlays for the prevention of pressure ulcers in patients admitted to hospital.

Design Cost effectiveness analysis carried out alongside the pressure relieving support surfaces (PRESSURE) trial; a multicentre UK based pragmatic randomised controlled trial. Setting 11 hospitals in six UK NHS trusts.

Participants Intention to treat population comprising 1971 participants.
\end{abstract}

Main outcome measures Kaplan Meier estimates of restricted mean time to development of pressure ulcers and total costs for treatment in hospital.

Results Alternating pressure mattresses were associated with lower overall costs ( 2283.6 per patient on average, 95\% confidence interval $-£ 377.59$ to $£ 976.79$ ) mainly due to reduced length of stay in hospital, and greater benefits (a delay in time to ulceration of 10.64 days on average, -24.40 to 3.09). The differences in health benefits and total costs for hospital stay between alternating pressure mattresses and alternating pressure overlays were not statistically significant; however, a cost effectiveness acceptability curve indicated that on average alternating pressure mattresses compared with alternating pressure overlays were associated with an $80 \%$ probability of being cost saving.

Conclusion Alternating pressure mattresses for the prevention of pressure ulcers are more likely to be cost effective and are more acceptable to patients than alternating pressure overlays.

\section{Introduction}

Older age, reduced mobility, impaired nutrition, vascular disease, faecal incontinence, and skin damage consistently emerge as risk factors for pressure ulcers. ${ }^{12}$ The annual cost to the UK NHS of implementing the current guidelines for the treatment of pressure ulcers was estimated in the region of $£ 1.4 \mathrm{~b}$ to $£ 2.1 \mathrm{~b}$ at 2000 prices. $^{3}$ Experts differ in their views as to the extent to which pressure ulceration is preventable ${ }^{4}$; however most would agree that pressure ulcers are often the consequence of poor preventive strategies. Between April 2002 and April 2004, the NHS Litigation Authority (www.nhsla.com/home.htm) received 56 claims for pressure damage (NHS Litigation Authority, personal communication, 2005), these included four instances where the patient died. The average cost of a claim was reported as £37 295 (\$70 453; €54 713) but ranged up to £375 000. Inadequate nursing care, lack of assistance and care, and failure or delay in diagnosis were often cited as causal. Early identification of vulnerable people and timely provision of a cost effective programme for the prevention of pressure ulceration in hospitals could prevent this cost to the NHS and the adverse health consequences of those affected.

Current National Institute for Health and Clinical Excellence guidelines on the prevention of pressure ulcers recommend the use of high specification foam mattress as the standard in vulnerable patients and surfaces such as alternating pressure mattresses for high risk people. ${ }^{5}$ This latter recommendation was based on expert opinion in the absence of research. Alternating pressure surfaces are available as whole mattress replacement systems (typically costing about $£ 4000$ ) and as overlays (typically $£ 1000$ ), which are placed on top of a standard hospital mattress. The relative costs and effects of these different systems are uncertain. We carried out an economic evaluation alongside a large multicentre pragmatic randomised controlled trial of alternating pressure mattresses and alternating pressure overlays. The clinical results of the trial are published in the accompanying paper. ${ }^{6}$

\section{Methods}

Full details of the trial's methods are available elsewhere. ${ }^{7}$ Briefly, eligible people were aged at least 55 years who were admitted to vascular, orthopaedic, medical, or care of the elderly wards, either as acute or elective admissions, in the previous 24 hours. Inclusion criteria were an expected length of stay of at least seven days, and an expected (if elective) or actual limitation of activities and mobility or an existing pressure ulcer of grade 2, or both. Trial completion was defined as improved mobility and activity, resolution of pressure ulcers for three consecutive days, transfer to non-participating wards or consultants, discharge from hospital, 60 days from randomisation, or death.

\section{Interventions}

Participants were randomised to receive an alternating pressure mattress or an alternating pressure overlay within 24 hours of hospital admission, using a remote telephone randomisation service providing concealed allocation. Rather than restrict the evaluation to prespecified products, we developed specifications for eligible surfaces to permit the hospitals to use mattresses and overlays from their existing stock or within their current rental contract (see Nixon et $\mathrm{al}^{6}$ ).

\section{Study design}

We carried out an economic evaluation analysis in the intention to treat population of the pressure relieving support surfaces 
Table 1 Description of unit costs associated with using alternating pressure mattresses or alternating pressure overlays for patients admitted to hospital

\begin{tabular}{|c|c|c|}
\hline Item & $\begin{array}{l}\text { Unit cost } \\
(£)\end{array}$ & Source \\
\hline \multicolumn{3}{|l|}{$\begin{array}{l}\text { Hospital costs by } \\
\text { specialty: }\end{array}$} \\
\hline $\begin{array}{l}\text { Care of elderly } \\
\text { people }\end{array}$ & 165.00 & $\begin{array}{l}\text { Health service database } 2003 \text {, Chartered Institute of } \\
\text { Public Finance and Accountancy }\end{array}$ \\
\hline Orthopaedic & 385.00 & $\begin{array}{l}\text { Health service database } 2003 \text {, Chartered Institute of } \\
\text { Public Finance and Accountancy }\end{array}$ \\
\hline Vascular & 374.00 & $\begin{array}{l}\text { Health service database 2003, Chartered Institute of } \\
\text { Public Finance and Accountancy }\end{array}$ \\
\hline \multicolumn{3}{|l|}{ Surface costs: } \\
\hline Overlay & 1010.50 & Huntleigh and Hill-Rom retail price list \\
\hline Mattress & 4173.60 & Huntleigh and Hill-Rom retail price list \\
\hline \multicolumn{3}{|l|}{ Daily cost of overlay: } \\
\hline 2 year life span & 1.38 & $\begin{array}{l}\text { Based on estimates of average unit cost provided by } \\
\text { manufacturers }\end{array}$ \\
\hline 5 year life span & 0.55 & $\begin{array}{l}\text { Based on estimates of average unit cost provided by } \\
\text { manufacturers }\end{array}$ \\
\hline 7 year life span & 0.40 & $\begin{array}{l}\text { Based on estimates of average unit cost provided by } \\
\text { manufacturers }\end{array}$ \\
\hline \multicolumn{3}{|c|}{ Daily cost of mattress: } \\
\hline 2 year life span & 5.71 & $\begin{array}{l}\text { Based on estimates of average unit cost provided by } \\
\text { manufacturers }\end{array}$ \\
\hline 5 year life span & 2.25 & $\begin{array}{l}\text { Based on estimates of average unit cost provided by } \\
\text { manufacturers }\end{array}$ \\
\hline 7 year life span & 1.63 & $\begin{array}{l}\text { Based on estimates of average unit cost provided by } \\
\text { manufacturers }\end{array}$ \\
\hline \multicolumn{3}{|l|}{$\begin{array}{l}\text { Daily rental of } \\
\text { mattress: }\end{array}$} \\
\hline Overlay & 8.23 & Huntleigh and Hill-Rom retail price list \\
\hline Replacement & 16.30 & Huntleigh and Hill-Rom retail price list \\
\hline
\end{tabular}

(PRESSURE) trial. ${ }^{6}$ We estimated the mean health benefits and mean total costs associated with both surfaces. ${ }^{89}$

\section{Data collection}

To estimate the number of pressure ulcer free days, we recorded the time taken for the development of a new grade 2 pressure ulcer for each patient. To estimate average length of stay in hospital, we routinely recorded the dates of entry and discharge from hospital for each patient. We did not record use of wound dressings as the clinical research nurses routinely observed patients with pressure ulcers up to grade 2 were not receiving wound dressings.

\section{Unit costs}

Table 1 provides a description of the unit costs. We calculated hospital treatment costs per day on the basis of estimates from the Chartered Institute of Public Finance and Accountancy of the cost per patient day in a care of elderly people, orthopaedic, or general surgery ward..$^{10}$ The unit purchasing and rental costs of each surface were estimated on the basis of UK retail prices provided by the manufacturers (Huntleigh Healthcare and HillRom). Patients were assumed to have remained on the allocated surface during their entire hospital stay. No robust evidence of the expected life span of the alternating pressure devices was available; however, clinical experts suggested that adequately used and maintained surfaces could last from two to seven years, provided they were not in constant use. For the base case analysis we assumed a two year life span. The pricing year was 2002-3.

\section{Heath outcomes}

We defined health benefit as the difference in the mean time to develop a pressure ulcer between the groups-that is, pressure ulcer free days. Survival analysis was used to calculate the restricted Kaplan Meier estimates of mean time to pressure ulcer development in each trial arm.
Table 2 Means (standard deviations) of cost estimates ( $£$ ) of using alternating pressure overlays or alternating pressure mattresses for the prevention of pressure ulcers in patients admitted to hospital

\begin{tabular}{lll} 
Item & \multicolumn{1}{c}{ Overlay } & Mattress \\
\hline \begin{tabular}{l} 
Resource use: \\
\hline Length of stay
\end{tabular} & $20.36(25.72)$ & \\
\hline Hospital costs: & & \\
\hline Base case & $6793.33(8196.52)$ & $6509.73(7347.56)$ \\
\hline Rental & $6967.81(8357.40)$ & $6747.48(7558.25)$ \\
\hline $\begin{array}{l}\text { Five year life span of } \\
\text { device }\end{array}$ & $6776.43(8177.07)$ & $6443.49(7278.95)$ \\
\hline $\begin{array}{l}\text { Seven year life span of } \\
\text { device }\end{array}$ & $6773.38(8173.56)$ & $6431.62(7266.67)$ \\
\hline
\end{tabular}

\section{Cost effectiveness analysis}

We carried out a cost effectiveness analysis, from the perspective of the UK NHS and Personal Social Service, on the intention to treat population for the period of hospital stay. ${ }^{11}$ The time horizon was shorter than one year-that is, the actual duration of hospital stay, consequently neither costs nor benefits were discounted.

To account for the skewed nature of data for costs and length of stay, we used generalised linear models to estimate the mean difference in overall hospital costs between the mattress and overlay groups. We used the Akaike Information criterion and the normal plot of deviance residuals to compare models assuming different distributions functions (Gaussian, gamma, inverse Gaussian) and link functions (identity, log). ${ }^{12}$ The final model assumed a gamma distribution for the data with an identity link function. ${ }^{13}$ The coefficient estimates of a generalised linear model with an identity link function represent the difference between groups using arithmetic means. To preserve the correlation between health benefits and overall hospital cost, we used non-parametric bootstrapping techniques to estimate the bias corrected $95 \%$ confidence intervals of the mean differences in time to development of pressure ulcers and costs between the overlay and mattress groups. ${ }^{14}$ We used Stata version 8 for all statistical analyses.

\section{Sensitivity analysis}

The robustness of the results was explored in three different scenarios: both devices assumed to be rented rather than purchased, a five year life span for both devices, and a seven year life span for both devices.

\section{Results}

A total of 1972 participants were randomised from 10 of the 11 hospitals participating in the randomised controlled trial comparing alternating pressure mattresses with alternating pressure overlays for the prevention of pressure ulcers. One patient who had been inadvertently randomised twice was excluded, leaving an intention to treat population of 1971 participants.

\section{Cost and health benefits}

In the base case analysis the generalised linear model which best described overall hospital costs indicated that on average the mattress group resulted in a mean reduction in total hospital cost of $£ 283.60$ (95\% confidence interval - £377.59 to $£ 976.79$, $\mathrm{P}=0.418$; table 2 ). This difference was not statistically significant.

The difference in Kaplan Meier restricted estimates of the mean time to development of pressure ulcers indicated that participants in the mattress group took 10.64 days longer to develop a pressure ulcer than participants in the overlay group (table 3). 
Table 3 Economic evaluation of alternating pressure overlays compared with alternating pressure mattresses for the prevention of pressure ulcers in patients admitted to hospital

Overlay-mattress

Base case analysis:

\begin{tabular}{lc}
\hline Differential health benefit (days) & $-10.64(-24.40$ to 3.09$)$ \\
\hline Differential cost $(£)$ & $283.60(-377.59$ to 976.79$)$ \\
\hline Sensitivity analyses: & $220.33(-459.85$ to 927.00$)$ \\
\hline Differential rental cost $(£)$ & $332.95(-325.27$ to 1020.77$)$ \\
\hline $\begin{array}{l}\text { Differential cost for five year life span } \\
(£)\end{array}$ & $341.76(-315.92$ to 1028.63$)$ \\
\hline $\begin{array}{l}\text { Differential cost for seven year life } \\
\text { span }(£)\end{array}$ &
\end{tabular}

This difference was not, however, statistically significant $(95 \%$ bias corrected confidence interval -24.40 to 3.09 days).

\section{Cost effectiveness analysis}

The base case economic analysis indicated that the mattresses are a dominant strategy when compared with the overlays; they are associated with a delay in the development of pressure ulcers and lower hospital costs. The sampling uncertainty associated with this result was investigated in an incremental cost effectiveness plane. Figure 1 shows the results of 1000 replicates of a non-parametric bootstrap of the estimated mean difference in cost and benefits. The majority of point estimates fall in the north west and south west quadrants of the cost effectiveness plane, suggesting that the overlays are associated with a more rapid development of pressure ulcers than are the mattresses.

Figure 2 is a cost effectiveness acceptability curve that represents the uncertainty associated with the decision to consider the mattresses a dominant alternative when compared with the overlays. The mattresses are associated with an $80 \%$ probability of being cost saving (the probability associated with a willingness to pay of zero) compared with the overlay. Similarly, a probability of about $15 \%$ was associated with overlays being cost effective for a range of willingness to pay values from $£ 25$ to $£ 30000$ for an extra pressure ulcer free day.

\section{Sensitivity analyses}

The results from the base case analysis were fairly robust to considering feasible variations in the life span of the pressure relieving surfaces or in changing provision between rental and purchase. The three sensitivity analyses in table 3 suggest that alternating pressure overlays are more costly and associated with a more rapid development of new grade 2 pressure ulcers than are alternating pressure mattresses.

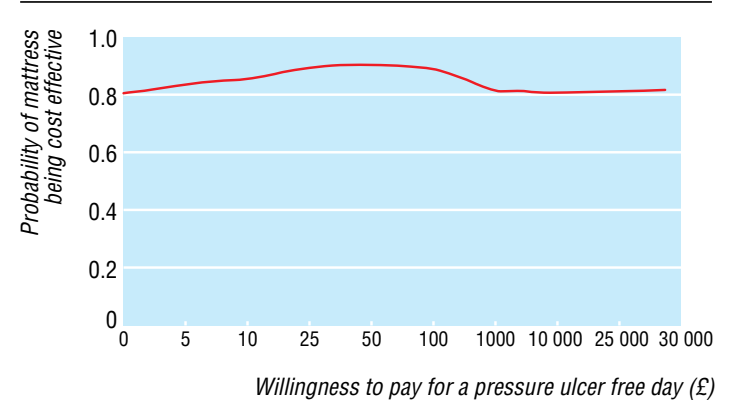

Fig 2 Cost effectiveness acceptability curve for alternating pressure mattresses

\section{Discussion}

Alternating pressure mattresses to prevent pressure ulceration in patients admitted to hospital are associated with lower costs and greater benefits and are more likely to be cost saving than alternating pressure overlays.

In this study we defined costs as mattress costs and hospital costs. During the trial we observed that grade 2 pressure ulcers did not usually receive dressings and other nursing care therefore we did not estimate for further treatment costs. ${ }^{7}$ The health benefits associated with the interventions were captured as pressure ulcer free days. We chose this approach rather than the more usual method of trying to capture a patient's utility through changes in quality of life because concurrent illness would dominate any quality of life measurement. Furthermore, the emphasis of our study was on prevention and participants who developed new ulcers were not followed-up until the ulcer healed. Although we did follow the healing of ulcers present at baseline, we did not have quality of life data that related to the time of ulceration (before admission) and therefore could not examine how quality of life changes with the status of pressure ulcers. The effect of pressure ulcer healing on people's health related quality of life may be the subject of future studies.

That the mattress is likely to be economically dominant may initially seem counterintuitive. The mattress has a higher purchase cost and no statistically significant effect on the proportion of patients developing an ulcer, the time to ulceration, the severity of ulcers, or length of stay. However, the purchase cost of these pressure relieving surfaces is low when viewed over their life span, and the difference in costs between overlays and mattresses is small over this time period. For a two year life span, the average cost per day would be $£ 1.38$ for an

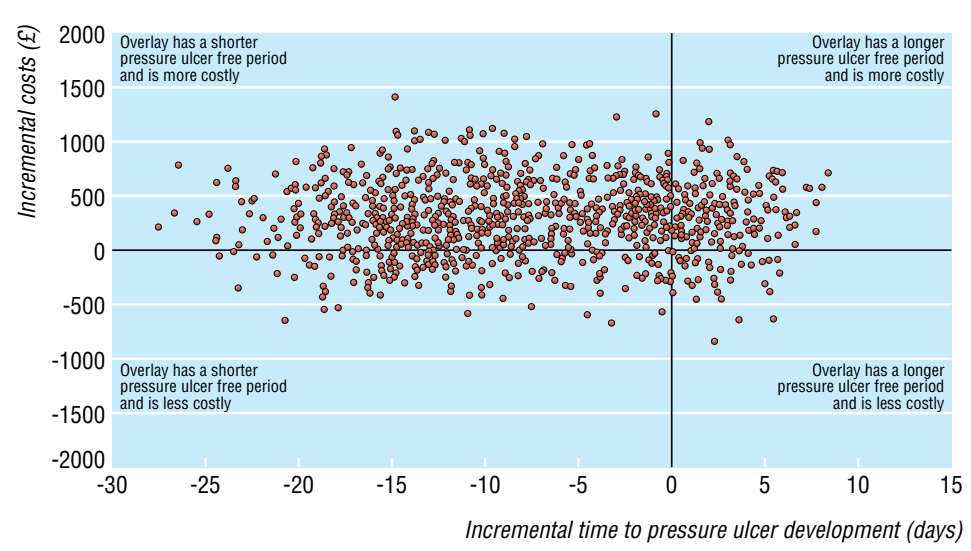

Fig 1 Incremental cost effectiveness plane of using alternating pressure overlays or alternating pressure mattresses for the prevention of pressure ulcers in patients admitted to hospital 
overlay and $£ 5.71$ for a mattress (table 1 ). This cost difference is small in the context of the daily inpatient treatment costs of $£ 165-£ 385$ (depending on specialty) and the reduced length of stay (on average, 1.22 of a day less) for mattress recipients in this trial and translates into an average reduction in costs of £283.6 per patient. The health benefit associated with the interventions was measured as the difference in mean time to develop a pressure ulcer, and also favoured the mattress (on average by 10.64 days). The accompanying clinical paper for this trial reports the median (rather than mean) time to ulceration, as is common practice owing to the skewed distribution of time to event data. ${ }^{6}$ By contrast, and as recommended for economic analyses, we use the mean time to pressure ulceration since the median would greatly underestimate the costs. ${ }^{8}$

The delay in ulceration associated with alternating pressure mattresses is crucial because the longer a patient avoids ulceration the less likely they are to go on to develop a pressure ulcer; a delay in ulceration allows the patient time to recover sufficiently from their acute episode so that their risk of ulceration recedes. We argue that although time to ulceration was a secondary end point in this trial, it should be considered as a primary end point in future studies since it is more informative economically and clinically. Furthermore, many of the risk factors for pressure ulceration in the participants do not completely disappear on discharge from an acute hospital (for example, impaired mobility), and simple proportions of pressure ulcers developed ignore the censored nature of these data.

Total cost is a function of length of hospital stay which itself is mainly a function of overall health status. Patients who are more ill are both more at risk of pressure ulcers ${ }^{15}$ and likely to have longer lengths of stay. It is probable that good nursing care, such as the provision of a support surface, will completely prevent pressure ulcers in a proportion of patients and will merely delay the appearance of ulcers in others. If the appearance of an ulcer is a proxy for acuity, and more acutely ill patients consume more healthcare resources, then delays in pressure ulceration suggest a potentially lower consumption of healthcare resources. The longer length of stay in patients with pressure ulcers is probably a consequence of comorbidities rather than the pressure ulcer itself. We found no effect when we tested to see whether the surfaces had a differential effect on length of stay depending on the presence of a pressure ulcer; overall lengths of stay for patients who developed a pressure ulcer on either surface were similar.

Since there are no robust estimates of the cost of a pressure ulcer we were not able to estimate the savings from pressure ulcers prevented. Such a costing study would need to be large, with a considerable length of follow-up, carried out in several centres to ensure generalisability, and would require careful observation of clinical practice in nursing care. It was not possible to undertake such a costing study within this trial.

Our results oppose those from the model based cost effectiveness analysis by Fleurence because data in that trial were based on expert opinion whereas we collected data within the trial. ${ }^{16}$ Furthermore, the previous model disregarded the fact that pressure ulcers are rarely the main reason for people being admitted to hospital. Thus the marginal cost of treating a pressure ulcer in hospital may be small compared with the overall costs of hospital treatment. In this sense our analysis reflects actual practice. Our assumption that participants remained on the allocated pressure relieving surface over their entire hospital stay is a conservative one; in reality patients are moved on to standard mattresses or higher specification surfaces after major changes in their risk of pressure ulcers. Given participants in the overlay group developed pressure ulcers earlier than those on the mattresses, this assumption will have over-estimated the cost of the mattresses, thus strengthening our conclusions.

Although neither the difference in mean overall hospital costs nor the difference in mean time to development of pressure ulcers between the two surfaces were statistically significant, decision makers need to make a decision. Failing to do so until statistically significant evidence becomes available would deny patients the possibility of experiencing the expected health benefits at an earlier stage and prevent the realisation of expected savings to the NHS. ${ }^{9}$ Although there was considerable uncertainty around the point estimates of mean health benefits and costs shown in table 3 , this should not result in large decision uncertainty for decision makers since even for large willingness to pay values (fig 2) the probability of the overlays being cost effective is only between $10 \%$ and $20 \% .{ }^{17}$

We thank the clinical research nurses Helen Barrow, Caroline Cooper, Fiona Corcoran, Patricia Hutchinson, Yvonne Meades, Dawn Parkes, Fiona Smith, Ann Fotheringham, Sarah Gowland, Ann Warriner, Caroline Smith, Richard Buckland, Monica Clark, Morag Doherty, Andrew Young, and Helen Marson; the participants and their relatives; ward nursing and medical staff of the six participating NHS trusts; and the independent trial steering committee: Jenny Hewison (chair), David Machin, and Gerben ter Riet. Contributors: NC, JN, AN, SM, and DT designed and initiated the trial. JN, $\mathrm{AP}$, and $\mathrm{HB}$ coordinated the trial. GC and $\mathrm{KH}$ analysed the clinical data. CI and DT analysed the economic data. All authors met regularly and contributed to trial management; all participated in the interpretation of results and approved the paper. NC is the guarantor.

Funding: UK Department of Health through its health technology assessment programme. The opinions and conclusions expressed here are those of the authors and do not necessarily reflect those of the UK NHS or the Department of Health.

Competing interests: JN has been reimbursed for attending conferences, has been paid speakers' fees, and received research funding from Huntleigh Healthcare.

Ethical approval: This study was approved by the North West multicentre research ethics committee and local ethics committees.

1 Reed RL, Hepburn K, Adelson R, Center B, McKnight P. Low serum albumin levels, confusion, and fecal incontinence: are these risk factors for pressure ulcers in mobilityimpaired hospitalized adults? Gerontology 2003;49:255-9.

2 Allman RM, Goode PS, Patrick MM, Burst N, Bartolucci AA. Pressure ulcer risk factors among hospitalized patients with activity limitation. JAMA 1995;273:865-70.

Bennett G, Dealey C, Posnett J. The cost of pressure ulcers in the UK. Age Ageing 2004;33:230-5.

4 Brandeis GH, Berlowitz DR, Katz P. Are pressure ulcers preventable? A survey of experts. Adv Skin Wound Care 2001:14:244, 245-4, 248.

5 National Institute for Health and Clinical Excellence. Pressure ulcer prevention: pressure ulcer risk assessment and prevention, including the use of pressure-relieving devices (beds, mattresses and overlays) for the prevention of pressure ulcers in primary and secondary care. NICE clinical guideline. 2003;7.

6 Nixon J, Cranny G, Iglesias C, Nelson EA, Hawkins K, Phillips A, et al. Randomised, controlled trial of alternating pressure mattresses compared with alternating pressure overlays for the prevention of pressure ulcers. BMJ 2006;332; doi=10.1136 bmj.38849.478299.7C.

7 Nixon J, Nelson EA, Cranny G, Iglesias C, Hawkins K, Cullum N, et al. Pressure relieving support surfaces: a randomised evaluation. Health Technol Assess 2006 (in press).

8 Thompson SG, Barber JA. How should cost data in pragmatic randomised trials be Thompson SG, Barber JA. How shoul
analysed? BMJ 2000;320:1197-200.

9 Claxton K. The irrelevance of inference: a decision-making approach to the stochastic evaluation of health care technologies.J Health Econ 1999;18:341-64.

\section{What is already known on this topic}

No previous trial based economic evaluation has compared alternating pressure mattresses with the less costly alternating pressure overlays

\section{What this study adds}

Alternating pressure mattresses were associated with lower costs and greater benefits and are more likely to be cost saving than alternating pressure overlays 
10 Chartered Institute of Public Finance and Accountancy. The health service database 2003. Croydon: CIPFA, 2003.

11 National Institute for Health and Clinical Excellence. Technical guidance for manufacturers and sponsors on making a submission to a technology appraisal. London: NICE, 2004.

12 Lindsey JK, Jones B. Choosing among generalized linear models applied to medical data. Stat Med 1998;17:59-68.

13 Barber JA, Thomson SG. Multiple regression of cost data: use of generalised linear models. J Health Serv Res Policy 2004;9:197-204.

14 Mihaylova B, Briggs A, Armitage J, Parish S, Gray A, Collins R. Cost-effectiveness of simvastatin in people at different levels of vascular disease risk: economic analysis of a randomised trial in 20,536 individuals. Lancet 2005;365:1779-85.

15 Baumgarten M, Margolis D, Berlin JA, Strom BL, Garino J, Kagan SH, et al. Risk factors for pressure ulcers among elderly hip fracture patients. Wound Repair Regen for pressure

16 Fleurence RL. Cost-effectiveness of pressure-relieving devices for the prevention and treatment of pressure ulcers. Int J Technol Assess Health Care 2005;21:334-41.

17 Fenwick E, O'Brien BJ, Briggs A. Cost-effectiveness acceptability curves-facts, fallacie and frequently asked questions. Health Econ 2004;13:405-15.

(Accepted 24 March 2006)

doi $10.1136 /$ bmj.38850.711435.7C
Department of Health Sciences, University of York, York YO10 5DD Cynthia Iglesias research fellow

David Torgerson professor

Nicky Cullum professor

Clinical Trials Research Unit, University of Leeds

Jane Nixon deputy director

Kim Hawkins assistant director (statistics)

Angela Phillips senior trial coordinator

$\mathrm{Su}$ Mason principal research fellow

Centre for Reviews and Dissemination, University of York

Gillian Cranny research fellow

School of Healthcare Studies, University of Leeds

E Andrea Nelson reader

Correspondence to: N Cullum nac2@york.ac.uk 